\title{
Auto-Align - Multi-Modality Fluorescence Microscopy Image Co-registration
}

\author{
William T. E. Pitkeathly ${ }^{1}$, Natalie S. Poulter ${ }^{2}$, \\ Ela Claridge ${ }^{3}$ and Joshua Z. Rappoport ${ }^{2, *}$ \\ ${ }^{1}$ Physical Sciences of Imaging in the Biomedical Sciences \\ (PSIBS) doctoral training centre, University of \\ Birmingham Edgbaston Birmingham, B15 2TT, UK \\ ${ }^{2}$ School of Biosciences, University of Birmingham, \\ Edgbaston Birmingham, B15 2TT, UK \\ ${ }^{3}$ School of Computer Science, University of Birmingham, \\ Edgbaston Birmingham, B15 2TT, UK \\ *Corresponding author: Joshua Z. Rappoport, \\ j.rappoport@bham.ac.uk
}

Multi-modality microscopes incorporate multiple microscopy techniques into one module, imaging through a common objective lens. Simultaneous or consecutive image acquisition of a single specimen, using multiple techniques, increases the amount of measurable information available. In order to benefit from each modality, it is necessary to accurately co-register data sets. Intrinsic differences in the image formation process employed by each modality result in images which possess different characteristics. In addition, as a result of using different measurement devices, images often differ in size and can suffer relative geometrical deformations including rotation, scale and translation, making registration a complex problem. Current methods generally rely on manual input and are therefore subject to human error. Here, we present an automated image registration tool for fluorescence microscopy. We show that it successfully registers images obtained via total internal reflection fluorescence (TIRF), or epi-fluorescence, and confocal microscopy. Furthermore, we provide several other applications including channel merging following image acquisition through an emission beam splitter, and lateral stage drift correction. We also discuss areas of membrane trafficking which could benefit from application of Auto-Align. Auto-Align is an essential item in the advanced microscopist's toolbox which can create a synergy of single or multi-modality image data.

Key words: fluorescence microscopy, image analysis, image registration, stage drift, time lapse

Received 10 June 2011, revised and accepted for publication 28 October 2011, uncorrected manuscript published online 1 November 2011

Fluorescence of organic compounds was first recognized in microscopy when scientists using ultra violet light noticed that light of longer wavelengths was emitted from the sample under investigation (1). This property, known as auto-fluorescence, drove the development of the first fluorescence microscope in 1911 (1). Future advances in fluorescent compounds, which could be introduced into a sample, massively increased the versatility of the technique as it was no longer dependent on the fluorescent properties of the inherent molecules present in a particular sample. The diversity of today's fluorescent markers combined with the current molecular and cellular techniques mean that almost any subcellular protein, pathway, process and organelle can be tagged $(2,3)$, and hence, imaged through fluorescence microscopy. A century on, fluorescence microscopy remains the method of choice for spatially locating specific molecules within live cells, and the desire to observe more specific cellular processes in situ has driven the development of numerous different fluorescence microscopy techniques. These employ different methods of illumination, as well as means of acquisition, in order to target specific regions of the sample. Each of these has its own advantages and disadvantages but currently there exists no technique that completely outperforms its alternatives. Multi-modality microscopes integrate two or more different techniques into one module which acquires data through the same objective lens, but use separate measurement devices. This allows for sequential and even simultaneous image acquisition, of the same sample, using multiple microscopes so that the advantage of using each technique can be included in the final analyses. To gain the benefits of each technique, their image data sets must be spatially coincident in order to be analysed.

Numerous applications in the field of membrane trafficking exist which would greatly benefit from correlative analysis of multi-modal data sets. Specifically, the combination of confocal and total internal reflection fluorescence (TIRF) microscopy would provide the benefits of each to be integrated, namely the ability to generate clear and bright images of specific intracellular focal planes with confocal, and the high signal to noise and ability to directly quantify motion at the plasma membrane in $x, y$ and $z$ with TIRF. Specific research areas where this would be beneficial include, but are not limited to:

Endocytosis: Uncoating of clathrin-coated vesicles does not usually occur within the TIRF field. This has been observed by combining TIRF and epi-fluorescence illumination (4-6). However, confocal has clear benefits over epi-fluorescence such as the ability to eliminate out-of-focus light using the confocal pinhole. Thus, the 


\section{Pitkeathly et al.}

combination of TIRF and confocal microscopy could provide a more complete analysis of these types of events.

Endocytic trafficking: The transfer of cargo and vesicular components between the cell surface and intracellular compartments could be analysed by combining TIRF and confocal microscopy. This would be possible for both endocytosis, tracking from the plasma membrane into endosomes, as well as for endocytic recycling, observing the recruitment of recycling endosomes within the cytosol and subsequent fusion with the plasma membrane. Furthermore, a combined analysis of endocytosis and endocytic trafficking could be performed using confocal microscopy to visualize the bulk transfer of membrane proteins from the cell surface to intracellular compartments such as lysosomes, and in the same samples, epi-fluorescence imaging to quantify whole-cell fluorescence in order to analyse receptor degradation following lysosomal sorting (7).

Post-Golgi trafficking: TIRF represents the gold standard for quantitative analysis of individual events of exocytosis. Although exceptionally sensitive for analysis of events at the plasma membrane, a major limitation of TIRF is that it cannot image deeper into the cell. Transfer of anterograde biosynthetic cargo out from the Golgi has been imaged through epi-fluorescence and other methods $(8,9)$. However, Golgi elements are generally not visible in TIRF. The addition of confocal images would allow spatial correlation of sites of vesicle fusion imaged in TIRF to the localization of the trans-Golgi network (TGN), as well as analysis of the trafficking and subsequent plasma membrane fusion of specific carriers which have budded from the TGN. One area in which this would be particularly useful would be the analysis of migrating cells which have been previously shown to demonstrate polarized Golgi localization and/or polarized sites of exocytosis (10-12). Thus, imaging the location of the TGN, the budding of vesicles from the TGN, the trafficking of these carriers through the cytosol using confocal microscopy and subsequent fusion with the plasma membrane with TIRF could be possible.

Microtubules: The microtubule cytoskeleton has been observed to extend into the TIRF field and trafficking and fusion with the plasma membrane of vesicles linked to these microtubules have been observed (12-14). However, imaging microtubule tracks in live cells by epi-fluorescence is very difficult. Thus, the ability to analyse microtubule motility in the same live cells by TIRF and confocal microscopy could permit the analysis of potential differences between the microtubules extending towards the plasma membrane and those deeper into the cytosol.

Actin: Many actin-based protrusions exist which can readily be imaged by TIRF. However, analysing how these correspond to cytosolic actin dynamics can be difficult.
The large density of actin in the cytosol makes epifluorescence imaging difficult, and the limited $Z$ resolution of TIRF suggests that employing confocal analysis in the same cells might generate novel insights into processes such as filopodia and lamellipodia assembly.

With these types of applications in mind, we have sought to develop a series of algorithms which can automatically align fluorescence microscopy images. The initial motivation behind the development of this software was registration of TIRF and confocal data sets; thus, the characteristics of the respective images hold the main influence behind the method of registration finally implemented. The different characteristics arise as a direct consequence of the image formation processes employed by each technique. Therefore, it is important to understand the image formation processes in order to recognize what the challenges are, from an image registration point of view.

\section{Microscopy Techniques - Image Generation}

The first epi-fluorescence microscopes evolved from the basic bright-field microscope with only the simple addition of a mercury light source and a set of strategically placed filters; the only further complication being the introduction of the fluorescent marker into the cell. Experiments targeting more specific intracellular processes required imaging modalities with the capability to visualize the subcellular components without the contaminating background fluorescence often present in epi-fluorescence images. Such demands led to the implementation of various other imaging techniques in cell biology. Examples of these include laser scanning techniques such as confocal $(15,16)$ and multi-photon techniques $(17,18)$, as well as TIRF microscopy $(19,20)$. Each of these has its own advantages and limitations due to the sample illumination and image collection methods employed.

Laser scanning methods, such as confocal microscopy, use a highly focused beam of laser light for sample illumination. Images are formed point by point from fluorescence confined to a small volume centred at the focal point of the objective lens that is typically measured using a photon multiplier tube (PMT). Any photons detected by the PMT have passed through a system of filters and a small pinhole onto which the filtered light is focused. The purpose of the pinhole is to reject light rays from out-of-focus planes to achieve 'optical sectioning' of a sample. Conventional confocal microscopes form two-dimensional (2D) images by scanning the focal point of the illumination beam in a series of line rasters in a lateral plane $(x-y$ plane) with respect to the face of the objective lens. The fluorescence detected by the PMT is recorded serially and the final image is constructed using a computer. Moving the sample in small axial increments through this lateral plane, obtaining a $2 \mathrm{D}$ image at each increment, allows the generation of 3D images (i.e. a confocal stack). The thickness of each optical section is 
dependent predominantly on the pinhole diameter as well as other factors such as the light wavelengths involved and numerical aperture of the objective (eqn 1) (21).

$$
\mathrm{FWHM}=\sqrt{\left(\frac{0.88 \cdot \lambda_{\mathrm{em}}}{n-\sqrt{n^{2}-\mathrm{NA}^{2}}}\right)^{2}+\left(\frac{\sqrt{2} \times n \cdot \mathrm{PH}}{\mathrm{NA}}\right)^{2}}
$$

The above equation shows the effective slice thickness of a $2 \mathrm{D}$ confocal image for a pinhole $\approx 1$ airy unit; where FWHM is the half width at full maximum of the image slice, $\lambda_{\mathrm{em}}$ the emission wavelength of the fluorescent signal, $n$ the refractive index of the emersion liquid, $\mathrm{PH}$ is the diameter of the pinhole and NA is the numerical aperture of the objective lens. A good confocal microscope is capable of forming image slices of volumes as thin as $\approx 1 \mu \mathrm{m}$ depending on the excitation wavelengths used $(21,22)$. Image acquisition times for these techniques are limited to the speed at which the line rastering can be performed, and thus the temporal resolution is sacrificed for an increased axial resolution. Although conventional confocal microscopes generally require $\sim 1$ second to construct an image with $512 \times 512$ pixels, high speed techniques such as spinning disc and resonant laser scanning confocal microscopes can acquire images much more rapidly (e.g. 15 times faster than confocal) $(16,23-25)$. Thus, these speeds are comparable to those of widefield techniques using a charge-coupled device (CCD) camera, although the field of view of the confocal is often smaller.

TIRF microscopy, as the names suggests, uses total internal reflection in order to produce an evanescent field subsequently used for sample illumination. Total internal reflection occurs when light propagating through a medium with refractive index, $n_{1}$, falls incident on a boundary with another medium that has a lower refractive index, $n_{2}$, at an angle of incidence equal to, or greater than, the critical angle, $\theta_{c}$, as defined by Snells law. The evanescent field produced at this boundary propagates parallel to the interface like a thin sheet and its intensity decays exponentially with perpendicular distance from the surface (eqn 2).

$$
I_{z}=I_{0} \exp \left(-\frac{z}{d}\right)
$$

The above equation describes the intensity profile as a function of distance, $z$, from the refractive boundary step; where $I_{0}$ is the intensity at distance $z=0$ and $d$ is the decay constant of the intensity. The value of $d$ depends predominantly on the ratio of refractive indices of the refractive media, as well as the excitation wavelength used (26). Microscopes using objectives with sufficiently high numerical aperture permit the TIRF illumination beams to be passed through the objective and onto the sample boundary at an angle above that of the critical angle $(26,27)$. For biological microscopy, this boundary is usually a glass coverslip $(n \approx 1.5)$ against some sample substrate $(n \approx 1.3)$, typically using visible laser light for illumination. Under these conditions, the effective penetration depth of the excitation field is generally $<100 \mathrm{~nm}$ above the coverslip (26). This makes the TIRF microscope ideal for imaging events occurring at or near the adherent plasma membrane. As the illumination is confined to the region just above the coverslip, the resulting images contain minimal levels of intracellular background fluorescence. TIRF is a widefield illumination technique so the fluorescence signal from the full field of illumination is collected in parallel using a CCD camera. This permits imaging at high temporal resolutions (e.g. tens of frames per second). The limiting factor of TIRF is that it is only capable of imaging one thin optical section just above the substrate on which the sample is adhered.

\section{Current Image Registration Procedures}

Co-registration of image data is a common problem in biomedical imaging and many methods have been developed to suit specific applications $(28,29)$. Many current methods for co-registration of multi-modality fluorescence microscopy images have relied heavily on human input. There are various commercial software packages which include: manual, semi-automated and automated alignment of generic microscopy image data. We will briefly review these methods and some of the software packages which include them.

\section{Manual Methods}

The most basic and least scientific approach to image alignment is a simple superimposition of one image over the other followed by manual manipulation through user controls of a computer. Images are considered to be registered when a better alignment cannot be achieved, based on visual inspection of the superimposed image. Dual-colour TIRF images have been aligned in this way using METAMORPH software (Universal Imaging) (30). Some groups introduce fiduciary markers, such as fluorescent beads, into their samples which are used to manually align the images $(31,32)$. The presence of definite reference points in both images increases reliability as it does not rely on the existence of any colocalization in the sample under investigation. Final accuracy of the alignment in this case relies on stationary fiduciary markers present in both images; thus, any movement between acquisitions of sequential images will cause inaccuracies. However, manual methods are prone to human error which will generally result in some inaccuracy of registration and they are also relatively time consuming.

\section{Semi-Automated and Automated Methods}

Nikon NIS elements (versions $3.1+$ ) have a registration tool based on manual landmark assignment (one or three points per image) in both images. This can correct for translation, rotation and scale when three points are selected. The human error from manual point selection 


\section{Pitkeathly et al.}

is reduced using the 'fine tune' option. This gives good results when landmarks are chosen accurately and 'fine tuning' is used. For some confocal/TIRF image pairs, choosing three definite common landmarks in both images can be difficult and also time consuming. Although fine tuning of the point selection is available, incorrect point selection can cause inaccurate registration.

Auto-Aligner by Bitplane SCIENTIFIC software provides both manual and automatic methods of microscopy image registration. It is capable of automatically aligning whole image stacks as well as individual images. Its automatic registration function accounts for translation and rotation but not scale. Images would have to be scaled accurately before the registration process to achieve accurate result; the precise scale relation between two image data sets is not always known and therefore not ideal for multimodality registration.

TurboReg is a non-commercial plugin for IMAGEJ for sub-pixel image registration. It implements a highly efficient optimization algorithm using a multilevel pyramid resolution scheme to maximize the speed of computation (33). The algorithm was initially designed for coregistration of functional biomedical image data sets (Positron Emission Tomography (PET) and functional Magnetic Resonance Imaging (fMRI)) but its application to various different data sets, including microscopy images, can be found in numerous publications $(34,35)$. The algorithm was designed as a fully automated registration system that can correct for rotation, translation and scale, but the IMAGEJ implementation (36) requires initial landmark identification before registration is performed. Although extremely fast, its application to multi-modality images, such as TIRF and confocal data sets, has potential problems due to the nature or the minimization similarity parameter used. Large relatively bright features are common in fluorescence microscopy studies for reasons such as heterogeneous expression levels of fluorescent proteins in a cell population. These are often omitted from an investigation but are commonly present in images that also contain more useful specimens. Because of the difference in illumination volume between TIRF and confocal microscopes, these features can appear far brighter in one data set (see the feature in the top left of Figure $1 A, B)$. TurboReg looks to minimize the difference squared between the images; therefore, a false registration can be achieved if the difference between a large bright feature and the feature of interest is smaller than the difference between the actual features of interest in both images.

\section{Auto-Align}

\section{Multi-modality microscopy image registration}

The main differences in the images from the two techniques arise as a result of the physical image formation processes briefly outlined in the discussion above (readers are referred to Refs $16,17,19-22,26,27$ for a more comprehensive description). If we assume that both microscopes image the same focal plane of the same fluorescent sample, through the same objective lens, then we expect to see some of the same information in both images. TIRF, because of its thin illumination depth, usually shows small (sub-diffraction limit) fluorescent objects with a higher contrast relative to the background. Confocal images usually contain greater levels of background fluorescence as the illumination thickness is greater than that of TIRF. If there are many fluorescent particles in the sample then the confocal image may also contain more features, again due to the greater optical section thickness (see Figure 2).

Further differences arise from the different hardware used to record the images. The line scanning nature of the conventional confocal microscope means that the spatial resolution can be varied whereas the TIRF resolution is generally fixed unless pixel binning is performed, in which case the resolution is reduced by a factor of $2^{n}$. In addition, the field of view of both microscopes can be varied: the scanning region of the confocal field can be changed either through cropping or zooming, and a different sub-set of pixels in the CCD array can be selected on the TIRF CCD camera. Because of these differences, images containing the same feature of interest can differ by up to three rigid transformational differences in the resulting images: (i) spatial scales of each pixel (the area each pixel physically represents), (ii) a translational offset (the relative distance of the centre of a feature to the centre of the image) and (iii) a rotational offset due to the misalignment of the horizontal pixel arrays in the CCD camera chip and the scanning lines of the confocal laser. Although (i) and (ii) can change every time the field of view is changed, the rotational offset (iii) should remain constant unless the orientation of the CCD is changed.

If we can assume that for TIRF and confocal image registration the rotational offset is always constant, it makes sense to determine its value accurately and then use it to correct images so that they differ only by translation and scale; corrections for these can then be addressed separately using slightly different techniques. Decomposing the problem in this manner will also reduce the time taken to calculate the difference in scale and translation as the complexity of the computation is reduced. With this reasoning, the problem can be broken down into two different parts: (i) the determination of the rotational offset and (ii) the determination of translation and scale using the calculated angle.

\section{Rotational offset: algorithm-1}

Auto-Align uses a separate algorithm to calculate the rotational offset between the scanning region of the confocal and the CCD camera array of the TIRF microscope. This algorithm uses an optimization process to calculate the rotational offset between a TIRF and confocal image pair given: an estimate of the scale 



Figure 1: Requirement from registration of images acquired from confocal and TIRF microscopes. The top row shows images of the same focal plane of HeLa cells expressing clathrin-GFP obtained through confocal (A) and TIRF (B) microscopy. The bottom row shows two overlay images, the confocal image in red and the TIRF in green. C) The geometrical centres of the confocal and TIRF images without any scaling or rotation. D) The registered images using Auto-Align, the confocal image is shown in red and the TIRF in green. The original TIRF image was scaled by 1.0813 and rotated by $1.07^{\circ}$ for the registration. Notice how the vesicles present in the centre cell have higher contrast in the TIRF image than the confocal image due to lower levels of background fluorescence. Also that the feature in the top left present in both images is very bright in the confocal image but does not stand out in the TIRF image. Scale bars represent $10 \mu \mathrm{m}$.

difference in the two images, an estimate for the rotational offset and errors associated with both of these values. The estimate angle reduces the computation time compared to that of searching over all angles. Determination of the estimate scale is calculated from the ratio of the lengths of a line joining the same two points in both images and an estimate angle is calculated based on the difference in angle of the same two lines with respect to the horizontal. The human error and subjective nature of this is dramatically reduced because the algorithm performs a search over the range of angles defined by the angle error provided. The assumption here is that the points chosen in both images do in fact correspond to the same features existing in both images and that realistic errors are provided (typically $\pm 1-2$ ).
Algorithm-1 copes well with the noise typically present in fluorescence microscopy images (Appendix S1 and Figure S3), but produces optimal results with images that have high contrast features and high signal to noise ratio. Although the algorithm will calculate this offset with a high degree of accuracy for a single pair of ideal images, several different image pairs from the same instrument should be used and the mean result is taken as the final rotational offset. This is to average out any variations due to small rotations caused by stage drift (see below).

Despite its optimization structure, this algorithm is by no means computationally efficient owing to the large number of image convolutions it performs. On a good desktop computer $(3 \mathrm{GHz}$ quad core, $4 \mathrm{G}$ of 



Figure 2: Images illustrating the difference in the illumination methods employed by confocal and TIRF microscopes on three fluorescent nanospheres $(\sim 100 \mathrm{~nm}$ in diameter) at the focal point of the same objective (neglecting diffraction). A) The confocal laser illuminates all three spheres as they all lie within the illumination volume near the focal point. B) The effective penetration depth of the evanescent field is $\sim 100-200 \mathrm{~nm}$ and its intensity reduces exponentially with distance from its origin, so only the closest two spheres are illuminated. C and D) show the difference in images of the same three nanospheres formed by confocal and TIRF microscope. In the confocal case, all there spheres are close to the focal point so are all illuminated with almost the same intensity and all are shown in the final image (C). Only two spheres are present in the TIRF image (D), the second only just visible due to the exponential profile of the evanescent field.

RAM), algorithm-1 will take approximately 30 seconds to calculate the final result on a pair of $512 \times 512$ pixel images. This time will vary from image pair to image pair and also if the initial guess is inaccurate and the search error is large. Performing this on multiple image pairs will take several minutes, but as mentioned above, the rotational offset only needs to be determined once, therefore accuracy is chosen over efficiency.

\section{Translation and scale: algorithm-2}

Algorithm-2 calculates the difference in image scale and final translation between the features in the images using the rotational offset calculated by algorithm-1. This algorithm requires the pixel calibration values (i.e. the resolution of the images) of both the TIRF and confocal images, along with the possible errors incurred when determining these vales. The algorithm uses a three-stage process to complete the registration: (i) calculates a rough translation between the images using the ratio of the pixel calibration values as an estimate image scale difference, (ii) performs a scale optimization test between the TIRF image and a local region of the confocal image located at the co-ordinates of the rough translation and (iii) calculates the final translation between the confocal image and the TIRF image scaled using the optimal scale calculated in the previous step. As the first stage is intended to determine a rough translation, it performs its calculation using smaller, down-sampled copies of the input images. The second step, scale testing, optimizes the scale difference from the initial estimate, first at a low resolution, and then again at a higher resolution. These calculations at reduced resolution decrease the overall computation time, while providing sufficient information for accurate calculation at high resolution. For large images, a sub-area of a TIRF image can be selected which is used for calculation and then the full images are registered; this is to further reduce computation time. Algorithm-2 copes well with the noise typically present in fluorescence microscopy images (Appendix S1 and Figures 3 and 4).

If fast sequential or simultaneous image acquisition is possible, this algorithm can be applied to dual TIRF and confocal time series experiments. This is particularly useful for imaging processes such as vesicle trafficking, receptor dynamics and focal adhesion disassembly.

\section{Other areas of fluorescence microscopy}

The algorithms presented above also have application to single-modality image data sets, such as time-lapse and simultaneous multi-colour imaging data, which must be spatially coincident in order to be analysed.

\section{Stage drift correction}

Stage drift occurs owing to small lateral movements of a microscope's sample stage over time. This becomes a particular problem when trying to analyse motion of objects within the sample as it is difficult to determine whether the change of position of an object relative to the edges of the field of view is due to sample activity or stage drift. If the morphology of the total sample does not change significantly between frames (i.e. $l_{i+1}(x, y) \approx$ $\left.l_{i}(x+\Delta x, y+\Delta y)\right)$, then performing normalized crosscorrelation (NCC) on adjacent time frames can be done to detect any motion in the image due to stage drift. If the net motion of the sample is significant between each frame (greater than that of the stage drift), then NCC will calculate the net motion of the sample between 




Figure 3: A basic schematic representation of the optics used to separate two wavelengths of light from a mixed fluorescence emission signal. Dual-colour imaging systems excite using two beams simultaneously. As a result, the fluorescence emitted from the sample consists of a mix range of wavelengths from two emission spectra. To split this mixed signal into two channels, the light has to be separated by wavelength and then measured using a CCD camera. The mixed signal enters the beam splitter at the front face; the light rays below a specific wavelength travel straight through the cube undeflected and the light rays with wavelengths above a specific wavelength are reflected by $90^{\circ}$. A prism reflects the reflected beam again by $90^{\circ}$ so that the two beams run parallel before being projected onto a CCD camera array.

frames. Correcting for the net motion like this can allow more precise measurement of objects moving within the sample. Because of the type of the image registration method used here, the algorithm will be less stable for image sequences containing several cells moving in different directions with significant movement between frames.

\section{Simultaneous dual wavelength image registration}

Some widefield microscopes can perform simultaneous dual wavelength imaging. Fluorescence emitted from the sample is collected through the same objective lens and then chromatically separated using a beam splitter. Images from both wavelengths of light are projected in adjacent positions onto a single CCD array (see Figure 3).

Using one CCD array means that the recorded image contains the fluorescence signal for both wavelengths of emission. In terms of registration of these signals, there are potentially three image transformations to consider: translation, scale and possibly a rotation. There is an obvious translation involved from the fact that the signals were separated by the beam splitter. Perhaps less obvious is the difference in scale. This may arise if the chromatic response of the magnification optics is not linear over the range of wavelengths used. Determining the colocalization of fluorescence in the two images requires that they are spatially registered. Some experiments may be based on two fluorescent markers that never colocalize within the sample. In this scenario, the methods used in the algorithms described above will fail. However, the relative position of the centre of the images with respect to the CCD should not change from experiment to experiment. Therefore, the registration parameters (i.e. translation, scales and rotation) can be pre-calculated through registration of images of fluorescent samples that emit light over a broad range of wavelengths. Thus, these registration parameters can then be saved and used to align any other images from this microscope.

\section{Results}

\section{Multi-modality image registration}

Auto-Align is capable of registering data from TIRF, epi-fluorescence and confocal microscopes (Figure 4). Auto-Aligns' algorithm-1 determined that the rotational offset between the CCD camera array and confocal scanning line rasters on the laboratory's multi-modality fluorescence microscope (Nikon A1-R Ti incorporates TIRF epi-fluorescence and confocal microscopes) is $1.07^{\circ}$ with a SD of $0.0913^{\circ}$. All the individual results were calculated in fewer than seven iterations and these had converged to a single value before reaching the maximum number of iterations (if the result has not converged before the maximum number of iterations, then it may just have a similar value by chance and therefore not an accurate result).

\section{Confocal and epi-fluorescence image registration}

Automated alignment of images from epi-fluorescence and confocal microscopes were achieved through the use of Auto-Aligns' algorithm-2. For images of the same cell obtained through the same $60 \times$ objective, the image scale difference was found to be 1.0771 . Therefore, scaling the epi-fluorescence image by this factor changes the image resolution to that of the confocal image. Figure $4 \mathrm{~B}$ shows a crop of the epi-fluorescence image of the same cell in the confocal image. Figures $4 \mathrm{~A}$ and $5 \mathrm{D}$ show a crop of the registered result through Auto-Align.

\section{Confocal and TIRF image registration}

Automated alignment of images from TIRF and confocal microscopes was achieved using a procedure identical to that used to align epi-fluorescence and confocal images. For images of the same cell obtained through the same $60 \times$ objective, the image scale difference was found to be 1.0813 . Figures 1 and $4 \mathrm{E}$ show the result of this.

\section{Application: transferrin uptake via clathrin-mediated endocytosis}

Internalization of transferrin occurs through clathrinmediated endocytosis. Imaging both clathrin and transferrin with TIRF shows high levels of colocalization at the adherent plasma membrane (Figure 6A,C). Confocal 


\section{Pitkeathly et al.}


Figure 4: A demonstration of Auto-Align's multi-modality capability. Panel (A) shows a cropped version of the confocal image shown in the Figure 1. The same cell is shown imaged in epi-fluorescence (B) and TIRF illumination (C). Panel (D) is the registration of the confocal and epi images and $(E)$ is the registration of the TIRF and confocal images (confocal is shown in red). Both the epi- and the TIRF images were rotated by $1.07^{\circ}$. The epi- image was scaled by a factor of 1.0771 and the TIRF by 1.0813 . One might expect that epiand the TIRF images have exactly the same pixel scale but slight differences in magnification can occur due to small difference in the light path to the CCD. The scale bar represents $9 \mu \mathrm{m}$. 



Figure 5: Use of Auto-Align to compensate from stage drift. A crop from a larger TIRF image of a HeLa cell expressing GFP-tagged $\beta 3$-integrin over a period of $45 \mathrm{~min}$. The top row of images shows data from the original image sequence. $A$ and $B)$ The first and the 45 th time-point in the series. Panel (C) shows the RGB merge of the two images, the (A) in red and the (B) in green; these images show little colocalization. Panels ( $E$ and $F$ ) in the second row show the same images as those above but have been corrected for stage drift. Panel (G) shows colocalization between the corrected images in the RGB merge of (E) and (F). Images (D) and (H) show average projection through all 45 images for the original and the corrected image data. The projection through the original data (D) has a blurred appearance; this is due to stationary objects relative to the cell being moved by stage drift relative to the field of view. The projection through the corrected data $(\mathrm{H})$ appears far less blurred as stationary objects have been aligned throughout the stack. Scale bars represent $10 \mu \mathrm{m}$.

imaging at the same focal plane (Figure 6D-F) permits coregistration with the TIRF data. A confocal stack through the cell aligned with the TIRF/bottom confocal image, reveals vesicles containing transferrin that have lost their clathrin coat. Figure 7B,D and F illustrates one section of the stack at $2.75 \mu \mathrm{m}$ into the cell where many vesicles containing transferrin, but not coated in clathrin are present. Alignment of TIRF images with confocal stacks taken rapidly through the same live sell will allow tracking of clathrin-coated vesicles containing cargo, such as transferrin, as they are endocytosed from the adherent membrane and move into the cell, eventually shedding their clathrin coat.

\section{Stage drift correction}

Auto-Align corrects for lateral stage drift of a time-lapse image sequence for images obtained every minute over a 45-min period using the Nikon A1 TIRF system. The first and final images of this time series are shown in Figure 5A,B; over the 45-min period there has been some focal adhesion activity, e.g. adhesion assembly. The overlay of these images (Figure $5 \mathrm{C}$ ) shows that they are not spatially coincident. Performing an average projection through all images in the time series gives an image with a blurred appearance (Figure 5D), suggesting random movement due to stage drift. Figure 5D shows an overlay of the first and final images in the sequence after Auto-Aligns' stage drift correction; this image shows far greater spatial colocalization than these same images prior to stage drift correction (Figure $5 \mathrm{C}$ ). Figure $5 \mathrm{G}$ shows the average projection of all the images in the sequence, in which this image has a far clearer appearance than that of Figure 5D and hence demonstrates that the effects of staged drift have been corrected for.

\section{Simultaneous dual wavelength image registration}

Auto-Align is capable of registering dual wavelength images obtained through a beam splitter as shown in Figure 7. It was found that the resolution of the green channel (the top feature in Figure 7A) was $0.5 \%$ greater than that of the red channel as the scale difference between the features in each channel was 1.005. Automatic translation of these images leaves them spatially coincident.

\section{Comparison of Auto-Align and TurboReg}

The ability of Auto-Align to register images from multimodality microscopes was tested against that of another IMAGEJ plugin TurboReg, mentioned earlier in the text. While TurboReg was able to successfully register some TIRF/confocal image pairs, it does fail to register others; Figure 8 is an example of this. Here, TIRF and confocal images of the same focal plane of the same sample were used to test the two registration methods (see 



Figure 6: Transferrin is internalized through clathrinmediated endocytosis. Scission of the vesicle from the plasma membrane allows the clathrin-coated vesicle to move from the membrane into the cell. Shortly after scission, a vesicle sheds its clathrin coat leaving only a lipid vesicle containing transferrin and associated receptors. TIRF images (A) and (C) show clathrin-dsRed and Transferrin-Alexa488 at the adherent plasma membrane. Image (E), an RGB merge of $(A)$ and $(C)$, shows colocalization between clathrin and transferrin. Images (B) and (D) show the same cell imaged using confocal microscopy focused at $2.75 \mu \mathrm{m}$ into the cell. Vesicles from the plasma membrane have usually shed their clathrin coat once they have reached this depth and as a result there is visibly less colocalization between clathrin and transferrin, shown in the merged image (F). Scale bars represent $10 \mu \mathrm{m}$.

Figure $8 \mathrm{~A}, \mathrm{~B})$. At first sight, the images appear to be registered but inspection of the finer details shows that Auto-Align (Figure 8C) achieves better alignment than TurboReg (Figure 8D). For cases when TurboReg does register the multi-modality images, it generally completes the registration in less time than Auto-Align, depending on various factors such as the reliability of the landmark selection used for TurboReg's initial alignment estimates of the errors on the values for image scales required for Auto-Align.

TurboReg has the benefit of being able to retrieve differences in image scale, rotation and translation in a


Figure 7: Use of Auto-Align to register dual colour images obtained through and emission beam splitter MDA-MB-231 cells expressing $\beta 3$-integrin-GFP and paxillin-RFP imaged using an Olympus TIRF system equipped with a dual wavelength beam splitter. The feature top of image (A) is $\beta 3$-integrin-GFP and the bottom is Paxillin-RFP. Image (B) shows an RGB registered image using Auto-Align; the $\beta 3$-integrin is shown in green and paxillin in red. The paxillin image was scaled by a factor of 1.005 and translated to align. Scale bars represent $8 \mu \mathrm{m}$.

single execution, whereas Auto-Align initially requires the rotational offset calculated using one programme before a second programme can perform a complete alignment. This two-stage process is initially more time consuming as the chosen method for determining the rotational offset by Auto-Align can take up to several minutes depending on the desired accuracy of the result. However, once this has been determined it becomes a known constant for further registration which is then performed in a matter of seconds (depending on image sizes, scale errors used, computer speed, etc.).

\section{Discussion and Summary}

The automated image registration tools detailed here provide a powerful means of maximizing the accuracy of quantitative data analysis of specific time-lapse experiments for single and multiple data sets. Automated 


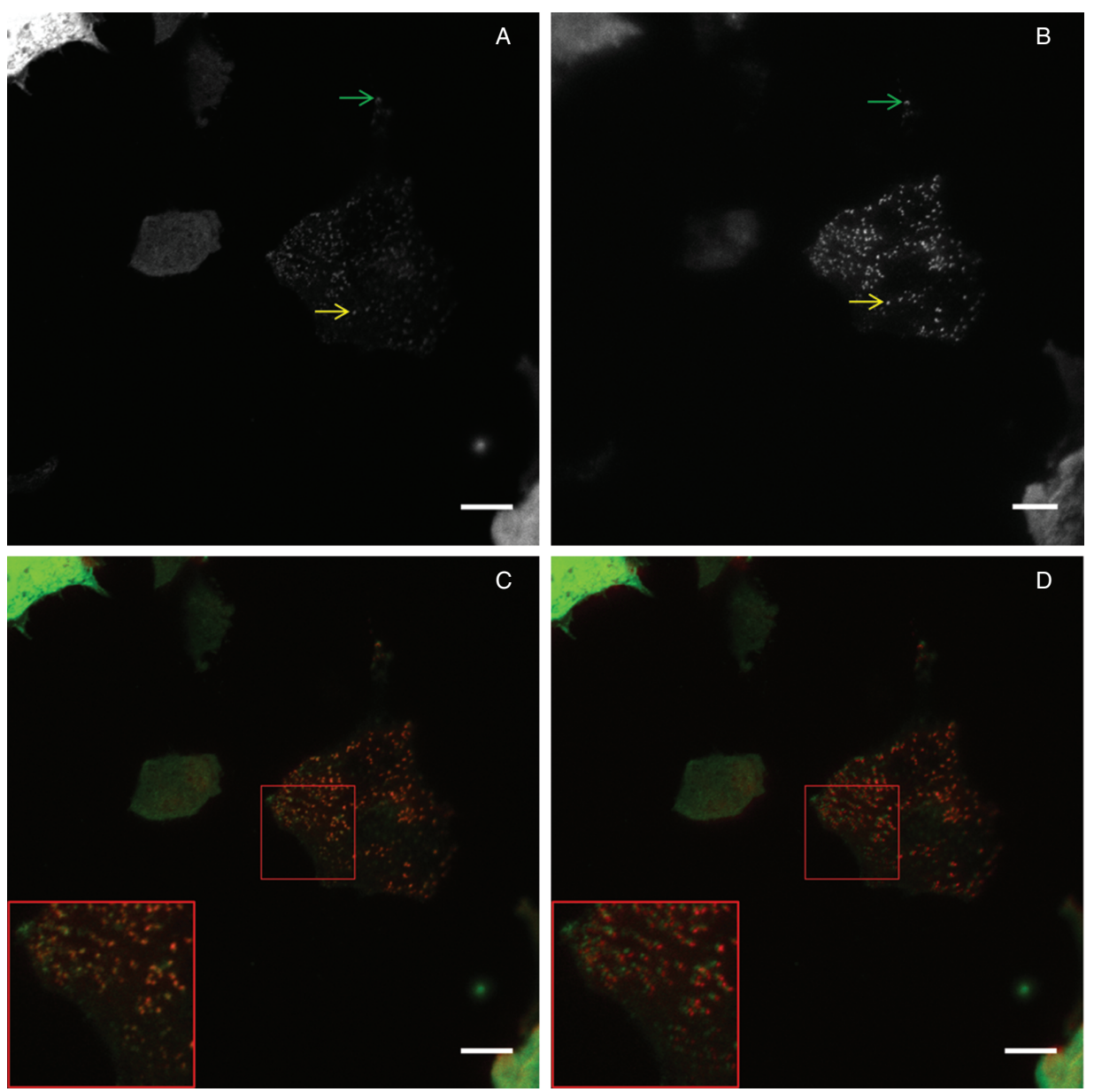

Figure 8: A TIRF and confocal image pair of the same sample in the same focal plane registered using Auto-Align and TurboReg. The arrows on images (A) and (B) point to the landmarks chosen to aid registration for TurboReg. Although almost all of the same features can be seen in both images, they appear to have different relative intensities with respect to the background. Images $(C)$ and (D) show (A) and (B) registered using Auto-Align (C) and TurboReg (D). The confocal images are shown in green and the TIRF in red. Visual inspection of both the registered images shows that Auto-Align has registered these images more accurately than TurboReg. Scale bars represent $12 \mu \mathrm{m}$.

multi-modality data synthesis is especially useful where multiple microscopy techniques can be used to image different aspects of the same sample simultaneously; this provides a more dynamic mixture of measurements to be included in the final analyses of a single experiment.

For the specific application of multi-modality image registration, we found that Auto-Align offered a more practical solution than TurboReg, as it was capable of registering multi-modality data more reliably on data typically found in membrane trafficking studies. With that said, TurboReg was designed for registration of functional medical images and not multi-modality microscopy images.

In experiments where intracellular movement in relatively stationary cells (relatively stationary between time frames) is of interest, then stage drift correction is absolutely necessary to derive accurate quantitative measurement of this intracellular movement; especially if the motion is very gradual with respect to the rate at which the images are acquired. For this stationary cell case, the stage drift correction method works well.

Although not presented here, Auto-Align provides further applications such as an indirect method for registration of confocal and bright-field images. Epi-fluorescence microscopes commonly share the same light path as bright-field microscopes and hence their images are already spatially coincident for the same field of view. Registration on an epi-fluorescence image to a confocal image will provide the registration parameters for the epi-fluorescences' bright-field counterpart.

Dual wavelength single CCD microscopy is becoming increasingly popular. Through registration of ideal samples, it is possible to determine precisely how the regions 
of the CCD, responsible for measuring signals from different channels, are spatially related. With this knowledge, it is possible to co-register data sets where colocalization is less obvious (or non-existent) with exactly the same accuracy as data sets with high levels of colocalization. The dual channel alignment here is far more accurate and consistent than manual channel alignment methods.

\section{Methods}

\section{Computational implementation}

All algorithms were implemented in JAVA as plugins for IMAGEJ $1.44+$. Algorithm-1 and algorithm-2 were also implemented in MATLAB where their performance was tested on a series of phantom data sets generated using models for the image formation processes of TIRF and confocal microscopes (for a comprehensive description of the algorithms implemented, see Appendix S1). The IMAGEJ plugin for Auto-Align is freely available from www.Rappoportlab.com.

\section{Image alignment, cell culture and microscopy TIRF-confocal and epi-fluorescence: confocal registration}

Before the images from the $C C D$ camera can be aligned with the images from the confocal microscope, the rotational offset between the confocal scanning line rasters had to be determined. Auto-Aligns' rotational offset algorithm was implemented on four image TIRF/confocal pairs. The image pairs for this were chosen because they had visibly low levels of noise and many obvious common features present in both images. Estimates for the angular offset, $\theta_{\text {est }}$, and scale difference, $s_{\text {est }}$, are determined using relative positions of user-selected landmarks in both images. The errors on these (used to define a search interval) were $\pm 2^{\circ}$ and $0.1 \cdot s_{\text {est }}$. The maximum number of iteration was set to 15 .

To fully register the TIRF and confocal data, the scale and translational differences in the images are required. These were calculated using AutoAligns' algorithm-2. The pixel sizes used for these TIRF and confocal images were 0.13 and $0.12 \mu \mathrm{m}$, respectively. Each of these was assigned a fractional error of 0.05 (or $5 \%$ ). The images used for the calculations were the first in focus slice closest to the coverslip showing transferrin at the plasma membrane along with the transferrin TIRF image.

The process for registering the epi- and confocal was identical to the TIRF and confocal.

\section{TIRF - confocal alignment: imaging clathrin and transferrin}

The angle of rotation used was $1.07^{\circ}$, calculated as described above. The scale and final translation of images were calculated using Auto-Aligns' algorithm-2. The pixel sizes used for these TIRF and confocal images were 0.14 and $0.14 \mu \mathrm{m}$, respectively. Each of these was assigned a fractional error of 0.05 (or $5 \%$ ). The images used for the calculations were the first in focus slice closest to the coverslip showing transferrin at the plasma membrane along with the transferrin TIRF image. Auto-Align scaled the TIRF images by a factor of 1.0266 and translated them to register (see Figure 6).

HeLa cells were plated into 35-mm cell culture dishes in DMEM supplemented with $10 \%$ foetal calf serum (FCS) and $1 \%$ penicillin/streptomycin. After $24 \mathrm{~h}$, when the cells were $\approx 80 \%$ confluent, the cells were transfected with clathrin-dsRed using Lipofectamine 2000 (Invitrogen) according to the manufacturer's instructions. After 24-h expression of clathrin-dsRed, the cells were split 1:3 and plated onto round glass coverslips. After a further $24 \mathrm{~h}$ (total of 48-h expression of clathrin-ds-Red), the cells were serum starved for $15 \mathrm{~min}$ at $37^{\circ} \mathrm{C}$ and then moved to $4^{\circ} \mathrm{C}$ where they were left to cool for $5 \mathrm{~min}$ and then incubated with transferrin-488 for a further $10 \mathrm{~min}$ at $4^{\circ} \mathrm{C}$. The cells were then moved back to $37^{\circ} \mathrm{C}$ for $15 \mathrm{~min}$ to allow endocytosis of transferrin to occur. The cells were then fixed in $4 \%$ formaldehyde and the coverslips were inserted into Bellco imaging chambers (BellcoGlass) and covered with PBS and imaged. Cells were imaged with a $60 \times$ objective (CFI TIRF Apo $60 \times$ oil NA 1.49, Nikon) using a Nikon-combined objective-based TIRF and confocal system set up on a Nikon EclipseTi inverted microscope. The 488-nm line of an Argon laser and the Green Diode $561 \mathrm{~nm}$ laser were used to image the transferrin and clathrin respectively. A TIRF image [captured on a 12-bit CCD camera (Ixon $1 \mathrm{M} \mathrm{EMCCD)]} \mathrm{was} \mathrm{taken} \mathrm{of} \mathrm{the} \mathrm{cells'} \mathrm{adherent} \mathrm{membrane} \mathrm{at} \mathrm{the}$ coverslip/cell interface, which was then set as the bottom focal plane of the cell for the confocal $z$-stack that was taken at $0.25 \mu \mathrm{m}$ steps using the Galvano scanner with sequential laser line acquisition (Figure 9).

\section{Stage drift correction}

Auto-Aligns' stage drift correction was used on a time series stack containing 45 frames.

HeLa cells were plated into 35-mm glass-bottom dishes (MatTek Corp) at a density of $6 \times 10^{5}$ cells per dish in DMEM supplemented with $10 \%$ FCS and $1 \%$ penicillin/streptomycin. After $24 \mathrm{~h}$, the cells were co-transfected with $\beta_{3}$-integrin-green fluorescent protein (GFP; a kind gift from Prof. Jonathan Jones, Northwestern University Medical School) and clathrin-ds-Red using Lipofectamine 2000 (Invitrogen) according to the manufacturer's instructions. Cells were then imaged $48 \mathrm{~h}$ after transfection with the Nikon A1-R Ti TIRF system using the 488-nm line of an Argon laser. Images were acquired every minute for a total of $45 \mathrm{~min}$. The TIRF image laser illumination was achieved through the microscope objective (CFI TIRF Apo 60× NA 1.49, Nikon) and images were captured on a 12-bit CCD camera (Ixon $1 \mathrm{M}$ EMCCD) using Nikon NIS ELEMENTS AR software.

\section{Dual-view acquisition of focal adhesion proteins}

Breast cancer MDA-MB-231 cells were plated into $35-\mathrm{mm}$ glass-bottom dishes (MatTek Corp) at a density of $6 \times 10^{5}$ cells per dish in DMEM supplemented with $10 \% \mathrm{FCS}$ and $1 \%$ penicillin/streptomycin. After $24 \mathrm{~h}$, the cells were co-transfected with $\beta_{3}$-integrin-GFP (a kind gift from Prof. Jonathan Jones, Northwestern University Medical School) and paxillin-red fluorescent protein (RFP; donated by Dr Maddy Parsons, Kings College) using Lipofectamine 2000 (Invitrogen) according to the manufacturer's instructions. After 48-h expression of the constructs, the cells were fixed in $4 \%$ formaldehyde and imaged in PBS on an Olympus TIRF microscopy system based around the Olympus IX81 ZDC microscope frame using a $150 \times$ objective (150x UAPON NA 1.45 objective, Olympus) and a dualview channel splitter (DV2-SYS dual view, equipped with a red/green filter). The $\beta_{3}$-GFP was excited using a 491-nm laser line and the paxillin-RFP was imaged using a 561-nm laser.

\section{Comparison with TurboReg}

Figure 8 shows a case were Auto-Align registers a TIRF and confocal image pair and TurboReg fails. Each programme was used in the following way.

\section{Auto-Align}

Prior to these images, the rotational offset between the CCD pixel array and the confocal line rasters was determined on a set of four image pairs obtained through the same microscope as described above. The rotational offset for this microscope set up as $1.07^{\circ}$. Auto-Aligns' multimodal image alignment (algorithm-2) was then used to register the images. The calibrated pixel sizes of the confocal and TIRF images were 0.12 and $0.14 \mu \mathrm{m}$, respectively. The search error fractional values on both of these were chosen as 0.1 (equal to $10 \%$ of their values). Auto-Align does not require the full TIRF image and can complete registration with only a cropped region containing the feature of interest. For this test, the full 



Figure 9: TIRF and confocal images of the same focal plane of HeLa cells expressing clathrin-dsRed and incubated with Transferrin-Alexa488 to illustrate transferrin uptake via clathrin-mediated endocytosis. Images (A), (B) and (C) show TransferrinAlexa488 (green). Images (D), (E) and (F) show clathrin-dsRed. Here, the TIRF images have been registered to the confocal images using Auto-Align. The TIRF images have been rotated $1.07^{\circ}$ and scaled by 1.0266 . The confocal images have been contrast adjusted so that the clathrin is more visible. Images $(C)$ and $(F)$ are the merge of the images $(A)$ and $(B)$ or $(D)$ and $(E)$, respectively, with the confocal shown in red and the TIRF in green. Scale bars represent $10 \mu \mathrm{m}$.

TIRF image was used so that both programmes were using the same data for registration.

\section{TurboReg}

TurboRegs' 'scaled rotation' registration mode was used as the method for registration as it accounts for scale, rotation and translation. The TIRF image was chosen as the 'source' and the confocal as the 'target' image. Landmarks corresponding to the same features in both images were carefully selected (see Figure $8 \mathrm{~A}, \mathrm{~B}$ ) and the quality of registration was set to 'accurate' before the automatic registration was selected.

\section{Acknowledgments}

The authors would like to thank EPSRC and BBSRC for funding: W. T. E. P. is funded through the EPSRC Physical Sciences of Imaging in the Biomedical Sciences (PSIBS) doctoral training centre, and N. S. P. and J. Z. R. are funded through BBSRC Grant [BB/H002308/1]. The confocal/TIRF microscope used in this research was obtained through Birmingham Science City Translational Medicine Clinical Research and Infrastructure Trials Platform, with support from Advantage West Midlands (AWM). The authors would also like to thank Jonathan P. Robinson and Hector A. Basevi, from PSIBS centre, for helpful comments.

\section{Supporting Information}

Additional Supporting Information may be found in the online version of this article:

Appendix S1: Auto-Align image co-registration.

Figure S1: The basic structure of the optimization algorithm designed to calculate the rotational offset between TIRF and confocal microscopy images. $I_{C}(x, y)$ and $I_{T}(x, y)$ are images from confocal and
TIRF microscopes, respectively, $s_{\text {est }}$ and $\theta_{\text {est }}$ are the current estimates for the scale difference and rotational offset between images and $s_{\text {opt }}$ and $\theta_{\mathrm{opt}}$ are the values giving the highest correlation from their respective tests. Scale is also optimized as precise knowledge of the scale is required in order to calculate the rotation precisely.

Figure S2: The basic structure of the algorithm used to determine the difference in translation between the TIRF and confocal images.

Figure S3: A graph showing the average calculated angle for 10 different phantom images rotated by $4^{\circ}$ and a scale difference of 1.1 containing different levels of noise. The signal to noise ratio (SNR) on this graph is that of only the TIRF images. Each corresponding confocal image has very similar noise levels. The error bars here represent the SEM of the calculated angles. Each colour series represents a different level of Gaussian additive noise and has seven increasing levels of Poisson noise.

Figure S4: The average scale calculated for the 10 phantom image pairs. The confocal images had a scale factor 1.1 greater than the TIRF images. Error bars represent the SEM. The signal to noise ratio (SNR) on this graph is that of only the TIRF images. The corresponding confocal images have very similar noise levels. Each colour series represents a different level of Gaussian additive noise and has increasing levels of Poisson noise.

Figure S5: A plot to show the translation between TIRF and confocal phantom image pairs calculated for different levels of noise. Prior to testing, the TIRF feature was translated by -20 pixels in both $x$ and $y$ directions. The SNR of the images is indicated by the colour of the data ring. The size of each ring indicates the number of points already with that value.

Video S1: shows movie images; the first is the original data set which moves due to stage drift, the second shows the same data set corrected for stage drift and the third shows the a RGB merge of the first two images. The RGB merge displays how much stage drift occurred over the 


\section{Pitkeathly et al.}

45 frames. In the red plane is the original data and the corrected in the green plane. The images are of the same cells shown in Figure 5 in the main article.

Please note: Wiley-Blackwell are not responsible for the content or functionality of any supporting materials supplied by the authors. Any queries (other than missing material) should be directed to the corresponding author for the article.

\section{References}

1. Rusk N. The fluorescence microscope. Nat Cell Biol 2009;11:S8-S9.

2. Prasher DC. Using GFP to see the light. Trends Genet 1995;11:320-323.

3. Zhang J, Campbell RE, Ting AY, Tsien RY. Creating new fluorescent probes for cell biology. Nat Rev Mol Cell Biol 2002;3:906-918.

4. Merrifield CJ, Perrais D, Zenisek D. Coupling between clathrincoated-pit invagination, cortactin recruitment, and membrane scission observed in live cells. Cell 2005;121:593-606.

5. Mattheyses AL, Atkinson CE, Simon SM. Imaging single endocytic events reveals diversity in clathrin, dynamin, and vesicle dynamics. Traffic 2011;12:1394-1406.

6. Saffarian S, Kirchhausen T. Differential evanescence nanometry: livecell fluorescence measurements with $10-\mathrm{nm}$ axial resolution on the plasma membrane. Biophys J 2008;94:2333-2342.

7. Mardakheh FK, Auciello G, Dafforn TR, Rappoport JZ, Heath JK. $\mathrm{Nbr} 1$ is a novel inhibitor of ligand-mediated receptor tyrosine kinase degradation. Mol Cell Biol 2010;30:5672.

8. Lippincott-Schwartz J, Roberts TH, Hirschberg K. Secretory protein trafficking and organelle dynamics in living cells 1. Annu Rev Cell Dev Biol 2000;16:557-589.

9. Hirschberg K, Phair RD, Lippincgtt-Schwartz J. Kinetic analysis of intracellular trafficking in single living cells with vesicular stomatitis virus protein G-green fluorescent protein hybrids. Methods Enzymol 2000;327:69-89.

10. Miller PM, Folkmann AW, Maia ARR, Efimova N, Efimov A, Kaverina I. Golgi-derived CLASP-dependent microtubules control Golgi organization and polarized trafficking in motile cells. Nat Cell Biol 2009;11:1069-1080.

11. Pouthas F, Girard P, Lecaudey V, Ly TBN, Gilmour D, Boulin C, Pepperkok R, Reynaud EG. In migrating cells, the Golgi complex and the position of the centrosome depend on geometrical constraints of the substratum. J Cell Sci 2008;121:2406.

12. Schmoranzer J, Kreitzer G, Simon SM. Migrating fibroblasts perform polarized, microtubule-dependent exocytosis towards the leading edge. J Cell Sci 2003;116:4513.

13. Toomre D, Keller P, White J, Olivo JC, Simons K. Dual-color visualization of trans-Golgi network to plasma membrane traffic along microtubules in living cells. J Cell Sci 1999;112:21.

14. Kreitzer G, Schmoranzer J, Low SH, Li X, Gan Y, Weimbs T, Simon SM, Rodriguez-Boulan E. Three-dimensional analysis of post-Golg carrier exocytosis in epithelial cells. Nat Cell Biol 2003;5:126-136.

15. Amos W, White J. How the confocal laser scanning microscope entered biological research. Biol Cell 2003;95:335-342.
16. Pawley JB. Handbook of Biological Confocal Microscopy. Springer Verlag; 2006.

17. Denk W, Strickler JH, Webb WW. Two-photon laser scanning fluorescence microscopy. Science 1990;248:73.

18. Diaspro A, Chirico G, Collini M. Two-photon fluorescence excitation and related techniques in biological microscopy. Q Rev Biophys 2005;38:97-166.

19. Axelrod D. Cell-substrate contacts illuminated by total internal reflection fluorescence. J Cell Biol 1981;89:141.

20. Mattheyses A, Simon S, Rappoport J. Imaging with total internal reflection fluorescence microscopy for the cell biologist. J Cell Sci 2010;123:3621.

21. Webb RH. Confocal optical microscopy. Rep Prog Phys 1996;59:427.

22. Müller M. Introduction to Confocal Fluorescence Microscopy: Society of Photo Optical. The International Society for Optical Engineering; 2006.

23. Tanaami T, Otsuki S, Tomosada N, Kosugi Y, Shimizu M, Ishida $\mathrm{H}$. High-speed 1-frame/ms scanning confocal microscope with a microlens and Nipkow disks. Appl Opt 2002;41:4704-4708.

24. Nakano A. Spinning-disk confocal microscopy - a cutting-edge tool for imaging of membrane traffic. Cell Struct Funct 2002;27:349-355.

25. Conchello JA, Lichtman JW. Optical sectioning microscopy. Nat Methods 2005;2:920-931.

26. Axelrod D. Total internal reflection fluorescence microscopy in cell biology. Traffic 2001;2:764-774.

27. Stout $A L$, Axelrod D. Evanescent field excitation of fluorescence by epi-illumination microscopy. Appl Opt 1989;28:5237-5242.

28. Maintz J, Viergever MA. A survey of medical image registration. Med Image Anal 1998;2:1-36.

29. Zitova B, Flusser J. Image registration methods: a survey. Image Vis Comput 2003;21:977-1000.

30. Rappoport JZ, Simon SM. Real-time analysis of clathrin-mediated endocytosis during cell migration. J Cell Sci 2003;116:847.

31. Moore EDW, Etter EF, Philipson KD, Carrington WA, Fogarty KE, Lifshitz LM, Fay FS. Coupling of the $\mathrm{Na}+/ \mathrm{Ca} 2+$ exchanger, $\mathrm{Na}+/ \mathrm{K}+$ pump and sarcoplasmic reticulum in smooth muscle. Nature 1993;365:657-660.

32. Meininger GA, Moore EDW, Schmidt DJ, Lifshitz LM, Fay FS. Distribution of active protein kinase $\mathrm{C}$ in smooth muscle. Biophys J 1999;77:973-984.

33. Thevenaz P, Ruttimann UE, Unser M. A pyramid approach to subpixel registration based on intensity. IEEE Trans Image Process 1998;7:27-41.

34. Rabut G, Ellenberg J. Photobleaching techniques to study mobility and molecular dynamics of proteins in live cells: FRAP, iFRAP, and FLIP. Live Cell Imaging: A Laboratory Manual, editors Robert D. Goldman and David L. Spector. Volume 1. 2005, 101-127.

35. Vicidomini G, Gagliani MC, Cortese K, Krieger J, Buescher P, Bianchini P, Boccacci P, Tacchetti C, Diaspro A. A novel approach for correlative light electron microscopy analysis. Microsc Res Tech 73:215-224.

36. Thevenaz P, Ruttimann UE, Unser M. A pyramid approach to subpixel registration based on intensity; 1998; http://bigwww. epfl.ch/publications/thevenaz9801.html. 